\title{
SIRIO: an integrated forest fire monitoring, detection and decision support system - performance and results of the installation in Sanremo (Italy)
}

\author{
A. Losso ${ }^{1}$, L. Corgnati ${ }^{1}$, S. Bertoldo ${ }^{1}$, M. Allegretti ${ }^{1}$, \\ R. Notarpietro ${ }^{1} \&$ G. Perona ${ }^{2}$ \\ ${ }^{1}$ Dipartimento di Elettronica e Telecomunicazioni, \\ Politecnico di Torino, Italy \\ ${ }^{2}$ CINFAI, Unità locale Politecnico di Torino, Italy
}

\begin{abstract}
Forest fires in our society cause a lot of damage, in particular regarding the economic and environmental landscape. In order to monitor a large portion of territory automatically, with a good cost/performances trade-off, it is necessary to develop new early warning systems. We propose a ground-based system with modular architecture, equipped with low cost commercial sensor. The idea is to develop the software able to manage forest fire monitoring. The technique is based on static and dynamic analysis of chromatic changes between images, tailored for our case of study in a large scale monitoring of vegetation and using different sensors to reduce or eliminate the false alarm rate. Concerning the image geo-referencing tool, the present work describes an innovative projective geo-referencing algorithm able to geo-reference complex orography regions using fixed ground station images. Besides, it does not need the collection of Ground Control Points, which is a very hard task in complex orography environments. In order to make a user oriented product and to help the operator during extinguishing activities, a decision support tool has been developed as well. This work presents the results of one year monitoring campaign conducted in cooperation with the Civil Protection Offices in Sanremo (IM), Italy.

Keywords: forest fires, smoke detection, geo-referencing, decision support, image processing, low cost commercial sensors.
\end{abstract}




\section{Introduction}

Forest fires in our society cause a lot of damage, in particular regarding the economic and environmental landscape. Forest fires have always been present in Italian territorial heritage, however, until the 60-70s (the period which dates back to the first statistics), the phenomenon has been kept within acceptable levels of impact in relation to the forest environment $[1,2]$. In figure 1 is represented the statistic behavior regarding forest fire occurrences and the surface burnt.

Forest Fire occurrences 1970-2010

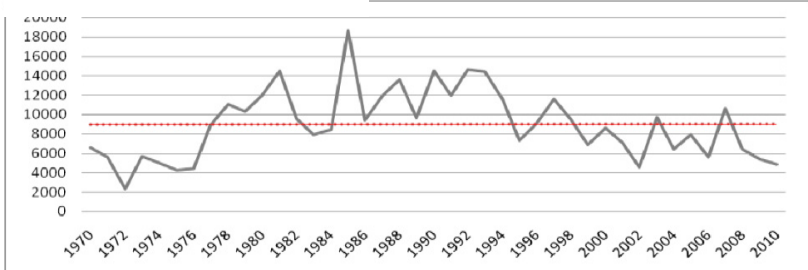

Surface burnt $1970-2010$

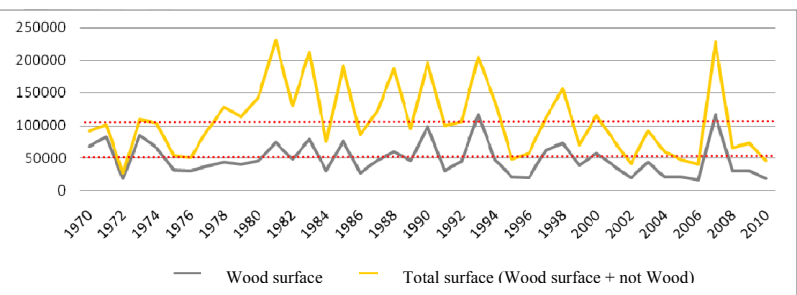

Figure 1: $\quad$ Forest fire behaviour in Italy from 1970 to 2010.

Despite the decreasing of forest fire occurrences from 1986, probably due to an increasing of prevention management, the wood surface burnt is more or less the same. In the Tuscany region, during 2011, the prevention costs were 11 Millions of Euro, equal to the same financing of 2010 [3]. Despite this, in 2011, fires occurred destroying 1.033 hectares. It was an increase of up to $66 \%$ with respect to the same season analyzed in 2010 [3, 4]. Prevention is not enough to contrast the deforestation caused by forest fires. According to our point of view, it is necessary to combine some important rules as: prediction, monitoring and early detection. The solution could be the studying and developing of new engineering instruments in order to face the problem. In particular, solutions must to be low-cost oriented in order to minimize the financing and maximize the results. A great number of low-cost oriented projects exist in the scientific landscape in order to contrast forest fire severity: tower watch, satellite monitoring, ground based sensors, etc.

Our project is based on a ground based system located far away from the area which we intend to monitor; for that reason it is not subject to damage caused by forest fires. The system, equipped with low-cost commercial sensors, is also suited for early warning monitoring and detection $24 / 24$ hours with a high spatial 
resolution. During the performance campaign (from September 2010 up to December 2012) there was a high reliability to the weather conditions.

The sensors generate images every few minutes and send them to the server where the algorithms work. Our technique (see section 3.2.1) is based on static and dynamic analysis of chromatic changes between images, as FernándezBerni et al. [5] proposed, tailored for our case study in large scale monitoring of vegetation and using different sensors to reduce or eliminate the false alarm rate.

Concerning the image geo-referencing tool, the present work describes an innovative projective geo-referencing algorithm able to geo-reference complex orography regions using fixed ground station images. In addition, it does not need the collection of Ground Control Points (Archetti et al. [6] and Archetti and Lamberti [7]), which is a very hard task in complex orography environments.

The system, named SIRIO, is developed by Remote Sensing Group (RSG) of Politecnico di Torino. An overview can be found in Corgnati et al. [8].

The forecast evaluation of the fire risk has an addressing function on surveillance and monitoring activities. The monitoring system operates with low cost optical sensors scanning Visible, Near Infrared and Thermal Infrared bands and a high precision low cost moving system. The data, coming from the sensors, are sent, through very a flexible communication system to the server station where the image interpretation algorithms work. In order to make a user oriented product and in order to help the operator in the extinguish operations, a decision support tool has been developed.

This tool allows us to add more information layers overlapped on a Google Maps $^{\circ}$. Information layers closer to the fire severity position as: helicopter landing spots, water point supply spots, access road etc. This tool allows us to add more information layers overlapped on a Google Maps ${ }^{\mathcal{O}}$. Information layers closer to the fire severity position as: helicopter landing spots, water point supply spots, access road etc.

\section{The system}

SIRIO system is divided into two main tasks: Fire risk Prevision and Forecasting, Hardware and Software Architecture.

The model developed by RSG group, FIRECAST ${ }^{\circledR}$, generates maps highlighting the areas where forest fire risk is high. The monitoring system will be placed and focused into the areas where the fire risk is relevant according to FIRECAST $^{\circledR}$ model (Corgnati et al. [9]).

\subsection{SIRIO system architecture}

The Architecture is divided into three main layers: Sensor and multi-frequency monitoring, Data Processing, User and decision support layer.

SIRIO optimizes technological, logistic and human resources in wildfires fight assuring high performances and maximum flexibility thanks to its modular architecture based on independent operative modules and on an embedded 


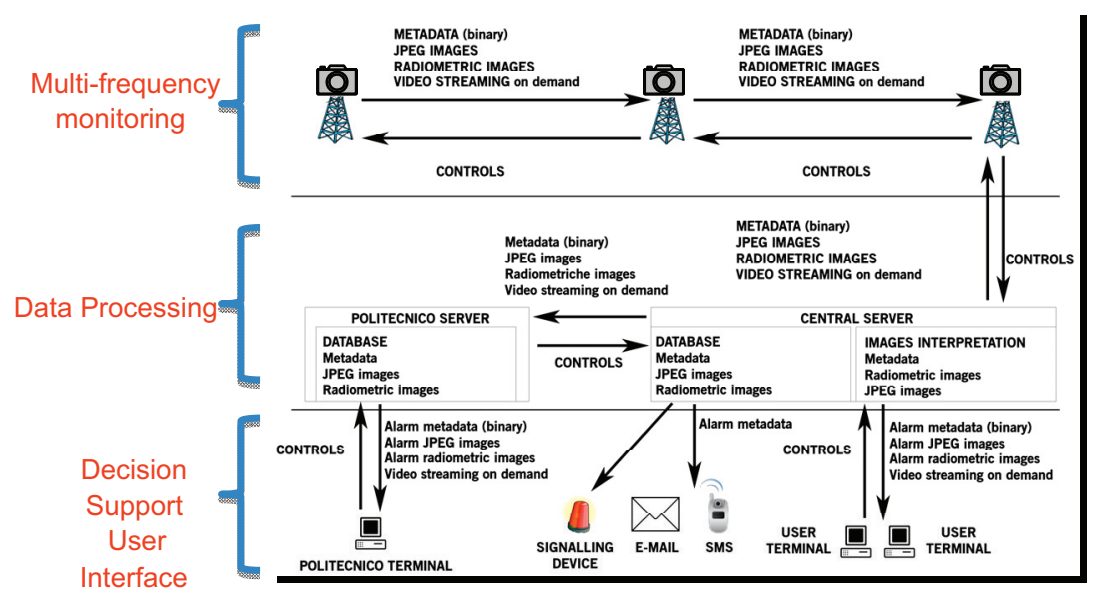

Figure 2: $\quad$ SIRIO system architecture.

communication system. The architecture is shown in figure 2 as proposed on Forest Fires II conferences (Corgnati et al. [8]).

The first layer is build-up by several systems located in those areas where the FIRECAST $^{\circledR}$ maps show the risk; they can communicate to each other system through internet, GPRS or WiFi connection. They are equipped with several sensors which can scan the whole monitoring area in several frequency bands. Each sensor is equipped with a computational module responsible for data acquisition integration. Data are transferred to the Data Processing layer where central server runs the image interpretation software (fire risk evaluation, smoke detection), the data storage, the alarms management and the decision support product generation. After the image interpretation, the central server sends confirmed alarms to the responsible agencies and operators as SMS messages, e-mail messages and activation of signalling devices. GUI interface are also developed in order to help the operator in case of fire severity. SIRIO is installed at Sanremo (IM), Italy, as test system. The test system is placed on the Civil Protection's roof fig from September 2010 up to December 2011. It generates data which are able to process to the data processing layer, where the image interpretation algorithms work. Once an alarm is found on an image, the software provides us to send an e-mail to the Civil Protection operators and to show the real time situation through a graphic user interface (GUI).

\section{The architecture}

\subsection{Sensors and multi-frequency monitoring}

The core of the system is made up by an engine sensors equipped, which scans the monitored scene. Monitoring scans and schedules, data acquisition, panoramic image composition and data transfer on central server are managed by the VM95 ${ }^{\circledR}$ controller. SIRIO can be equipped with several sensors due to the 
the VM95 ${ }^{\circledR}$ controller. SIRIO can be equipped with several sensors due to the modular architecture. The most important sensors that could be installed are: Canon Powershot A 640 photo camera, Sony Block video camera, Canon EOS 400D reflex photo camera, FLIR A40M thermal camera. SIRIO operates a multispectral scan in Visible (VIS), Near Infrared (NIR) and Thermal Infrared band (TIR).

\subsection{Data processing}

\subsubsection{Smoke detection algorithm}

In order to monitor a large portion of territory automatically, with a good cost/performances trade-off, it is necessary to develop new early warning systems. In particular, we propose an innovative method operating within an integrated surveillance system located in Italian alpine region. Concerning early warning frameworks, the presence of smoke, and therefore its early detection, is crucial as it is now the first reminder or warning that an outbreak is about to degenerate. In many cases, the flame may not be easily seen and not detected by hot-spot detection algorithm, as for example the burning of underbrush. In order to achieve low false alarm and missed detection rate, the results are processed by smoke detection system which evaluates images in visible domain.

This algorithm examines chromaticity changes and spatial and temporal patterns in the monitored scene. Every single pixel on images in the visible domain is composed of three components: Red (R), G (Green) and B (Blue). For the detection of smoke arising among vegetation, it proposes a more dataefficient option based only on the detection of sudden increases in the $\mathrm{B}$ component with respect to the background. The use of the B component is owing to its greater sensitivity to the changes generated by smoke in areas in which the vegetation is predominant, when compared to the $\mathrm{R}$ and $\mathrm{G}$ components and even to the combined luminance [5]. For that reason the smoke detection algorithm is developed which is able to recognize the $\mathrm{B}$ increasing between two consecutive images. In order to detect the sudden irruption of smoke in the images, the algorithm is composed of two blocks performed by two different modules.

The first block, called 'Static', identifies possible plumes of smoke rising from an outbreak with a scan of the chromatic pixel of captured images compared with a reference image. Defining $I b_{r}(i, j)$ as the intensity of the B component at the bin $(i, j)$ of the reference image and $I b_{c}(i, j)$ as the intensity of the B component at the bin $(i, j)$ of the current image, the condition to consider the $\operatorname{bin}(i, j)$ as "smoked bin" is :

$$
I b_{c}(i, j)-I b_{r}(i, j) \geq \frac{p}{100}\left(I b_{\max }-I b_{\min }\right)
$$

where $\mathrm{p}$ represent the percentage of B component set by $\left(I b_{\max }-I b_{\min }\right), I b_{\max }$ is the maximum value of the $\operatorname{bin}(i, j)$ in the current panoramic and $I b_{\min }$ is the minimum value of the $\operatorname{bin}(i, j)$ in the reference panoramic. Equation (1) is fulfilled if the $\operatorname{bin}(i, j)$ is covered by smoke. The sensitivity represents the value of percentage $\mathrm{p}$ set a priori by operators. It is necessary to set a $\mathrm{p}$ value in order to obtain the best optimal trade-off between detection and false alarm. 
The second block, called 'Dynamic', processes the images labelled with one or more alarm pixels by the Static block output and through spatial and temporal correlations isolates effective smoke plumes from other moving features (birds, moving tree due to wind, airplanes, clouds, shadows, etc.), thus reducing false alarms that may occur at the first stage of the process. If the smoke is detected, the system sends an alarm message.

\subsubsection{Geo-referencing image tool}

The smoke detection algorithm provides to elaborate the images in order to detect the possibly alarm which can occur on the monitored scene. At this point we have developed an algorithm which is able to help the operator in case of fire severity. It provides to give some important help instruments in term of additional layers support for the extinguish operations focusing on the forest fires event with a precise spatial location. The spatial location is given by the forest fires location evaluated through geometrical and projective transformation on the image elaborated. This transformation are able to link every pixel, or bin, of the images, with a latitude and longitude coordinates, in particular those affected by fire or smoke. Since the forest fires coordinates are found, the algorithms automatically provides to detect some important additional support layer as: water point supply, access-way, helicopter landing and operation squad locations. In particular, highlight those are close to the fire severity. The algorithm (Losso et al. [10]), is build up according image geometrical and projective transformation using the Digital Elevation Model (DEM) with a resolution of $100 \mathrm{~m}$. The operation is automatic and needs only some input at the beginning, no ground control point are taken a priori to geo-reference the images. The algorithm works for just one time, in the calibration phase, in order to generate a matrix within the geographical positions. This matrix is elaborated by a decision support algorithms which analyze the results from smoke detection and geo-referencing tool. Decision support methods also needs additional information layers which are overlap on a DEM, or on Google Maps ${ }^{\circ}$, in order to obtain which are more close to the fire event.

\subsubsection{Decision support and User Interface (GUI)}

The final step of our forest fires system is the user layer that is the final product user oriented developed according to the operators mandatory. That means, SIRIO system is tailored for every situation and operations as the orography, the environment, the position and the sensitivity order to set.

A Graphic User Interface (GUI), figure 3, was developed in order to help the operator and to give an early warning message, as e-mail or SMS, in case the algorithms detect a fire severity. In case of fire, a decision support tool show on a Google Maps ${ }^{\odot}$ the fire position and the support layers position as well. 


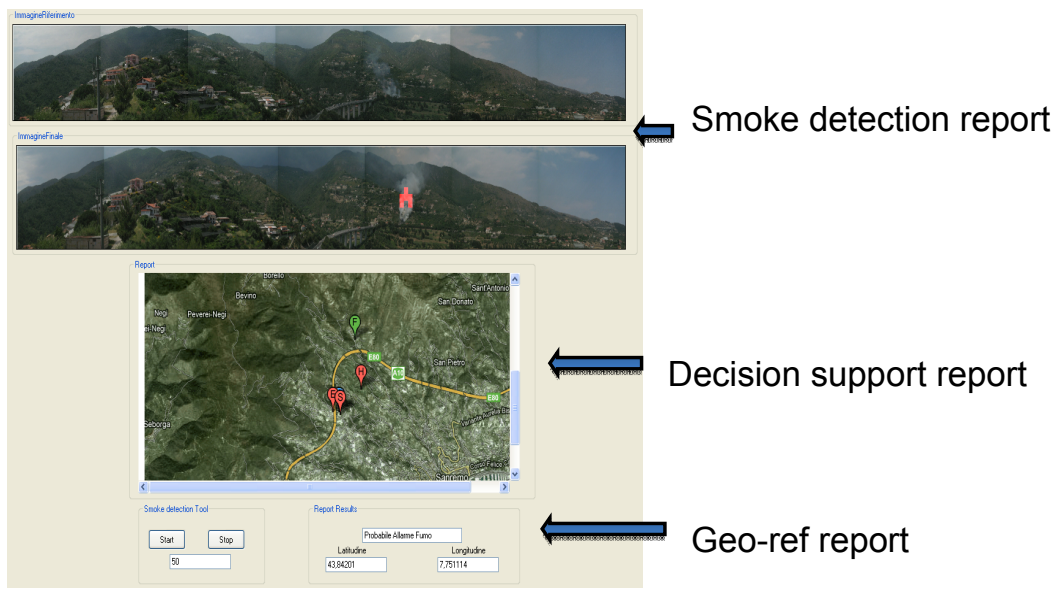

Figure 3: $\quad$ SIRIO GUI.

The support layers show the position of the Water Point supply, Helicopter Landing, Roads, Access Ways and Squad locations. It is very useful because it provides us to know the initial plan strategy to adopt for extinguishing the forest fires.

The GUI, through a decision support algorithm, show the operator which layers are closer to the event of fire and give a geographical positions on the Google map as well as the fire latitude and longitude. From figure 3, in case of fire severity, it is possible to see the layers automatically placed into the map in the correct geographic location. Label $\mathrm{F}$ is the position of the Fire, $\mathrm{H}$ is the Water point supply, $\mathrm{S}$ is the Squad location and $\mathrm{E}$ is the Helicopter landing. From Google Maps $^{(}$it is easier to see and understand the most common roads and access ways to the fire severity.

\section{Results}

This section presents and discusses the results coming out from the algorithm developed; in particular those coming from smoke detection algorithm and image geo-referencing tool. The system, as mentioned before, is located in Sanremo (IM), Italy, and it is fixed on the Civil Protection's building roof. The monitored area is the Mediterranean-Alpine orography in the Western-Liguria near to the France border. The portion monitored is rich with Mediterranean maquis vegetation and rural environment. From September 2010 up to December 2011 SIRIO has collected a large amount of data in the form of visible images from the Canon Powershot. The results generated depend on the timing of the VM95 ${ }^{\circledR}$ controller and on the portion of vegetation to be monitored. In our case the results are generated every 3 minutes. 


\subsection{Smoke detection results}

In order to validate the smoke detection algorithm, we used the contingency tables and the Relative Operation Characteristic curve (ROC). Some important indexes, calculated from contingency table 1, as probability of detection (POD), probability of false detection (POFD), false alarm rate (FAR) and accuracy are taken into account as well.

Table 1: (a) Indexes results $p=10$; (b) indexes results $p=30$; (c) indexes results $\mathrm{p}=50 ;(\mathrm{d})$ indexes results $\mathrm{p}=70$.

\begin{tabular}{|c|c|c|c|}
\hline \multicolumn{4}{|c|}{ Sensitivity $\mathbf{1 0}$} \\
\hline POD & POFD & FAR & Accuracy \\
\hline $\mathbf{0 . 8 7}$ & 0.035 & 0.32 & 0.96 \\
\hline
\end{tabular}

(a)

\begin{tabular}{|c|c|c|c|}
\hline \multicolumn{4}{|c|}{ Sensitivity $\mathbf{3 0}$} \\
\hline POD & POFD & FAR & Accuracy \\
\hline $\mathbf{0 . 7 8}$ & 0.026 & 0.28 & 0.95 \\
\hline
\end{tabular}

(b)

\begin{tabular}{|c|c|c|c|}
\hline \multicolumn{4}{|c|}{ Sensitivity $\mathbf{5 0}$} \\
\hline POD & POFD & FAR & Accuracy \\
\hline $\mathbf{0 . 6 7}$ & 0.018 & 0.24 & 0.95 \\
\hline
\end{tabular}

(c)

\begin{tabular}{|c|c|c|c|}
\hline \multicolumn{4}{|c|}{ Sensitivity $=\mathbf{7 0}$} \\
\hline POD & POFD & FAR & Accuracy \\
\hline $\mathbf{0 . 5 4}$ & 0.009 & 0.16 & 0.95 \\
\hline
\end{tabular}

(d)

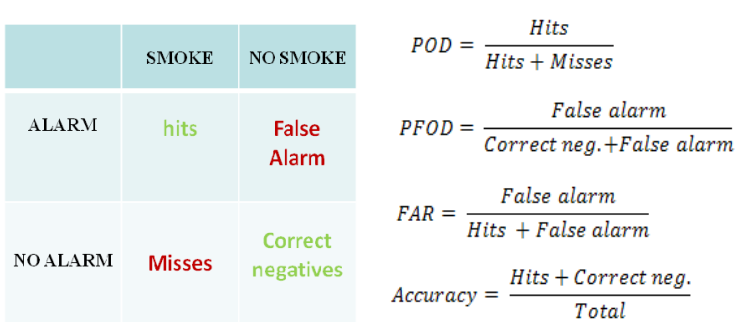

(a) (b)

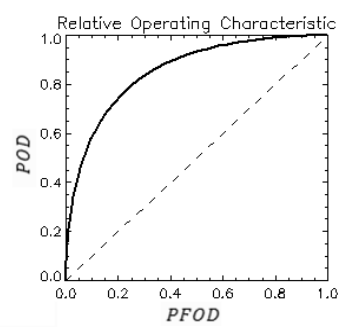

(c)

Figure 4: (a) Contingency Table, (b) Indexes, (c) ROC curve.

The final purpose is generating the ROC curve, changing the sensitivity $p$ (see section 3.2.1) from a minimum of 10 to 70 . The curve will be an important graphical method to understand the best optimal sensitivity trade-off. The 
sensitivity changing is done to find the optimal threshold to set into the algorithm for the Sanremo monitoring. The sensitivity represents how the algorithm is sensitive to the $\mathrm{B}$ changing between two images, the reference one and the current image. A great sensitivity detects less real alarm and more missed detection but could detect less false alarm as well. A small sensitivity detects more alarm then great sensitivity that could introduce a bigger false alarm rate.

Using the indexes it is not easily understood whether the sensitivity changing is better than others, according to the ROC curve the behaviour is plotted in figure 5 and the best optimal trade-off is when the curve tends to POD $=1$ and POFD tends to 0 .

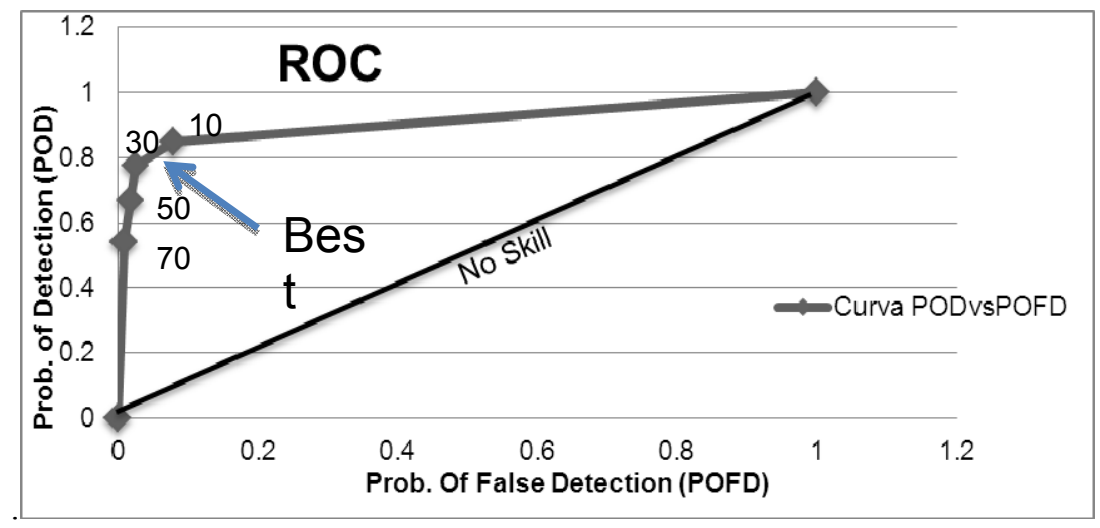

Figure 5: Smoke detection results plotted on ROC curve.

The figure shows the best value between $p=10$ and $p=30$, since for the system located in Sanremo the value $p=20$ should be the best to set in the parameter settings.

\subsection{Geo-referencing results}

The geo-referencing algorithm generates a geo-referred matrix with dimension $\mathrm{NxM}$, where $\mathrm{N}$ are the rows and $\mathrm{M}$ are the columns of the image. In order to validate the algorithm it is necessary to measure some point defined as real on the monitored scenario. The real points are taken detecting some visual features into the image. Using a GPS receiver and/or Google Earth ${ }^{\circ}$ application some of the real points have been measured and compared with those obtained in the same position on the matrix. This point are taken in proximity of visual features on the scene; using GPS or Google Earth ${ }^{\odot}$, we measure the latitude and longitude for each point and considering the Matrix it is calculated the differences, in the same visual point, between real point (measured with GPS and Google Earth ${ }^{\circ}$ ) and matrix point (calculated with the algorithm). It is also analyzed the differences in term of meters and plotted into a graph through a histogram.

Due to the depth of images, it is important to note that the pixel has a different size and area between foreground and background pixel. This is 
possible because the matrix is obtained using projective and geometric transformation of image through a DEM. Therefore, the differences between the real and calculated point may not be the same behaviour at every distance. For this reason, a validation threshold has been introduced. The validation is done using threshold at $1.5 \mathrm{~km}$ and $2 \mathrm{~km}$ from the camera location.

Graphs in figure 6 show that using different threshold the results are going to have a better behaviour because the pixel closer to the sensor are less subject to errors. The index which is able to show the behaviour is the Standard Deviation, remember that it is the value within are concentrated the $68 \%$ of the measures. Considering No threshold, the standard deviation is, respectively, $248 \mathrm{~m}$ latitude and $231 \mathrm{~m}$ longitude. If we consider a threshold at $2 \mathrm{~km}$ from the sensors, the value of Standard Deviation is going to be better than without threshold in both
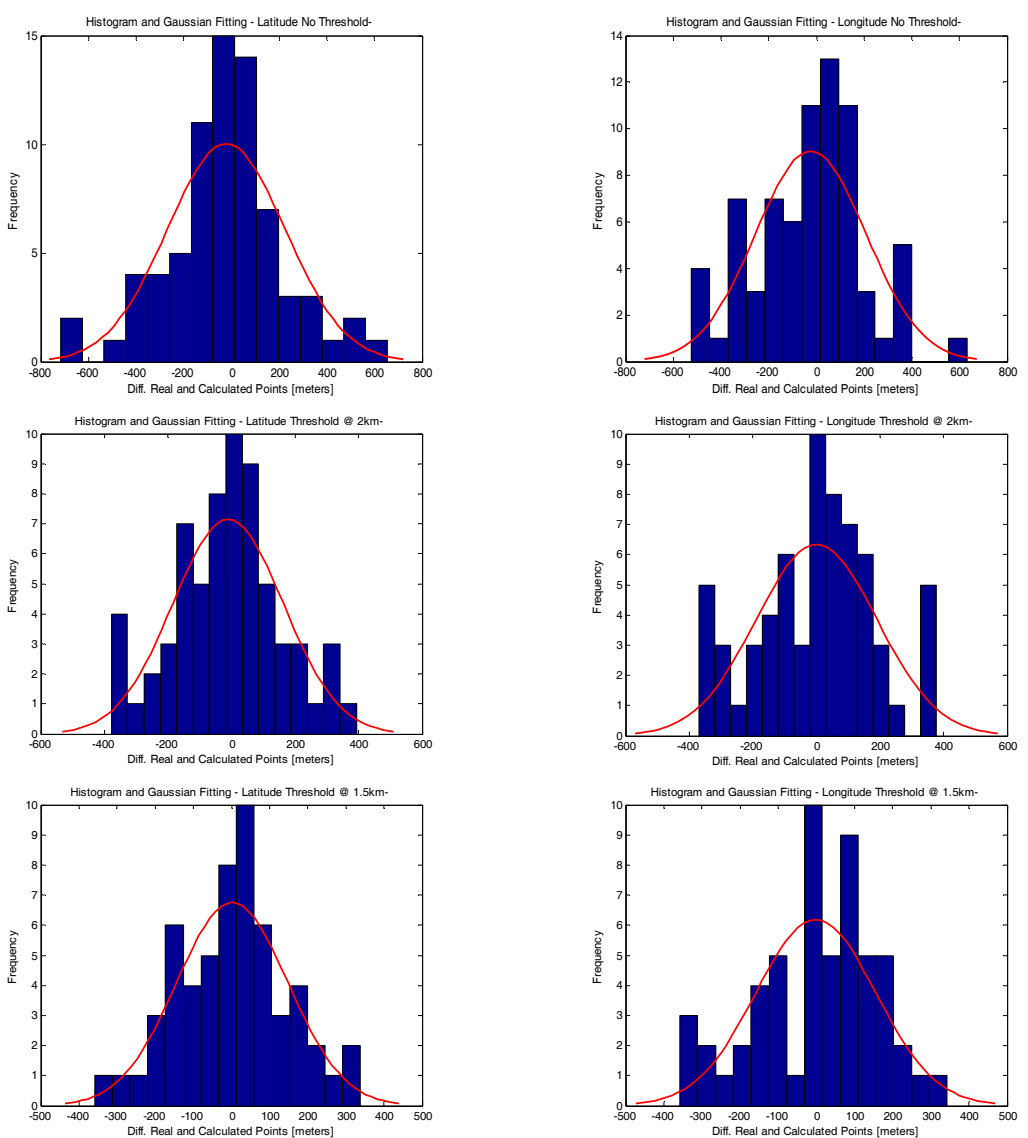

Figure 6: Latitude at right and longitude at left respectively for no threshold, $2 \mathrm{~km}$ and $1.5 \mathrm{~km}$ threshold. 
cases, latitude and longitude. The standard deviation is equal to $173 \mathrm{~m}$ and $190 \mathrm{~m}$. It is possible to see the tail disappearing.

The last threshold is set at $1.5 \mathrm{~km}$, and also in this case we obtain a better behaviour respect to the previous threshold. As a matter of fact, the index which show the behaviour, the Standard Deviation, is: $145 \mathrm{~m}$ for the latitude and $150 \mathrm{~m}$ for the longitude that means an error in the order of one DEM pixel.

The figures show us a histogram related to the frequency of the error introduced. The error is the difference between the real point measured by GPS or Google Earth and the calculated point found by the geo-referencing algorithm. The Gaussian in red, is a fitting curve of histogram and show a qualitative behaviour of the result, in this case, the error.

\section{Conclusion and future works}

SIRIO integrated system has been tested over different monitoring sessions and test areas and it is now operative in Liguria for wildfire monitoring and early warning. SIRIO achieves high performances in reliability, robustness flexibility, cost and consumption. From September 2010 up to now it provides a great reliability to the environmental condition for every season (Autumn, Winter, Spring and Summer). SIRIO shows us a great flexibility due to modular architecture: the system is able to work with all the sensors and algorithms or using one algorithm and sensor at a time. This allows the operators to use it setting the system according to the boundary conditions such as weather, orography, land cover, season, etc. Even, the modular architecture provides reliability in terms of power consumption and cost.

The price of the system is directly dependent on how many commercial sensors and algorithms are equipped and developed for the system. For this reasons, the system is low-cost oriented suited for complex orography and for every land cover condition.

It guarantees accurate smoke identification and produces geo-referenced information sets very useful for an effective decision support activities.

Just for resume the results, smoke detection algorithm has an accuracy (Hits + Correct Negative) equal to $96 \%$ and in the georef. tool results, the standard deviation show us an error in the order of one DEM pixel. The system has also enhanced territory monitoring and intervention planning for the operators.

The SIRIO system is under continuous development by the Remote Sensing Group of Politecnico di Torino; at present a new false alarm reduction tool based on combined VIS/NIR images is in the process of being implemented. Near Infrared images (NIR) are used for a new develop study. The main idea for the future is to develop more algorithms which run independently from each other, giving a certain degree of freedom in correlation with the cost and performance trade-off.

Concerning our results we can say that in order to reduce smoke detection error and false alarm rate, a frequency combination of images (NIR, TIR and VIS) could reduce the indexes POFD and FAR. 
In order to improve the geo-referencing performance, a more detailed DEM with higher resolution is needed. As much as the DEM resolution is high, a better result, in term of precision, will be found but the cost will obviously increase.

\section{References}

[1] Ricerca Forestale, www.ricercaforestale.it

[2] Osservatorio Incendi boschivi, www.incendiboschivi.org

[3] www.toscana-notizie.it

[4] www.corpoforestaledellostato.it

[5] Fernández-Berni, J., Carmona-Galán, R., Carranza-González, L., A visionbased monitoring system for very early automatic detection of forest fires, Institute of Microelectronics of Seville - Centro Nacional de Microelectrónica Consejo Superior de Investigaciones Científicas y Universidad de Sevilla, 2008.

[6] Archetti R., Torricelli E., Erdman R., Lamberti A., First application of a new imaging system for the coastal monitoring, Bologna.

[7] Archetti R. and Lamberti A., Studio dell'evoluzione di una spiaggia protetta da opera a cresta bassa mediante videomonitoraggio.

[8] L. Corgnati, A. Losso, G. Perona, SIRIO high performance Decision Support System for wildfires fight in alpine regions: an integrated system for risk forecasting and monitoring, Forest Fires II international Conference, Kos, Greece, 2010.

[9] Corgnati L., Gabella M., Perona G., FIREcast system - Previsional fire danger index computation system for alpine regions. 1st International Conference on Forest Fires 2008, 17-19 September 2008, Toledo (Spain).

[10] A. Losso, L. Corgnati, G. Perona, Innovative image geo-referencing tool for decision support in wildfires fight, Forest Fires II International Conference, Kos, Greece, 2010. 